\title{
Developing residential wireless sensor networks for ECG healthcare monitoring
}

Article

Accepted Version

Dey, N., Ashour, A. S., Shi, F., Fong, S. J. and Sherratt, R. S. (2017) Developing residential wireless sensor networks for ECG healthcare monitoring. IEEE Transactions on Consumer Electronics, 63 (4). pp. 442-449. ISSN 0098-3063 doi: https://doi.org/10.1109/TCE.2017.015063 Available at https://centaur.reading.ac.uk/73898/

It is advisable to refer to the publisher's version if you intend to cite from the work. See Guidance on citing.

Published version at: http://ieeexplore.ieee.org/document/8246822

To link to this article DOI: http://dx.doi.org/10.1109/TCE.2017.015063

Publisher: IEEE

All outputs in CentAUR are protected by Intellectual Property Rights law, including copyright law. Copyright and IPR is retained by the creators or other copyright holders. Terms and conditions for use of this material are defined in the End User Agreement.

www.reading.ac.uk/centaur

\section{CentAUR}

Central Archive at the University of Reading 
Reading's research outputs online 


\section{Full Text Version}

Title: Developing Residential Wireless Sensor Networks for ECG Healthcare Monitoring

Authors: Nilanjan Dey,

Department of Information Technology, Techno India College of Technology, West Bengal, 740000, India

e-mail: neelanjan.dey@gmail.com

Amira S. Ashour,

Department of Electronics and Electrical Communications Engineering, Faculty of Engineering, Tanta University, Egypt

e-mail: amirasashour@yahoo.com

Fuqian Shi, Senior Member, IEEE,

College of Information \& Engineering, Wenzhou Medical University, Wenzhou, 325035, PR China

e-mail: sfq@wmu.edu.cn

Simon James Fong,

Department of Computer and Information Science Data Analytics and Collaborative

Computing Laboratory University of Macau, Taipa, Macau SAR

e-mail: ccfong@umac.mo

R. Simon Sherratt, Fellow, IEEE

Department of Biomedical Engineering, the University of Reading,

RG6 6AY, UK

e-mail: sherratt@ieee.org

Publication: IEEE Transactions on Consumer Electronics

Publisher: IEEE

Volume: $\quad 63$

Issue: $\quad 4$

Date: $\quad$ Accepted 16 November 2017

pp.: $\quad$ not yet assigned

DOI: not yet assigned 


\section{Abstract}

Wireless technology development has increased rapidly due to it's convenience and cost effectiveness compared to wired applications, particularly considering the advantages offered by Wireless Sensor Network (WSN) based applications. Such applications exist in several domains including healthcare, medical, industrial and home automation. In the present study, a home-based wireless ECG monitoring system using Zigbee technology is considered. Such systems can be useful for monitoring people in their own home as well as for periodic monitoring by physicians for appropriate healthcare, allowing people to live in their home for longer. Health monitoring systems can continuously monitor many physiological signals and offer further analysis and interpretation. The characteristics and drawbacks of these systems may affect the wearer's mobility during monitoring the vital signs. Real-time monitoring systems record, measure, and monitor the heart electrical activity while maintaining the consumer's comfort. Zigbee devices can offer low-power, small size, and a low-cost suitable solution for monitoring the ECG signal in the home, but such systems are often designed in isolation, with no consideration of existing home control networks and smart home solutions. The present study offers a state of the art review and then introduces the main concepts and contents of the wireless ECG monitoring systems. In addition, models of the ECG signal and the power consumption formulas are highlighted. Challenges and future perspectives are also reported. The paper concludes that such mass-market health monitoring systems will only be prevalent when implemented together with home environmental monitoring and control systems.

\section{Index Terms}

Zigbee, electrocardiography, electrocardiogram, monitoring, wireless communication, transceivers, ECG monitoring system

\section{INTRODUCTION}

Electrocardiogram (ECG) is a vital diagnosis device to detect heart disease abnormalities. Cardiovascular disease affects the cardiovascular system, specifically the blood vessels and the heart. Strokes and heart attacks are the most common cardiovascular diseases in the general public that require continuous monitoring. ECG is the most broadly used cardiovascular disease monitoring technique that measures the electrical activities of the heart. An ECG system is a non-invasive monitor for evaluating the heart electrical activity, for measuring the regularity/rate of heartbeats, and for identifying any damage to the heart. The ECG includes the placement of electrodes on the human body surface at convenient places. Such electrodes are linked to the ECG monitoring device via cables to detect and amplify the electrical impulses of the heart, where heart conditions change the ECG characteristic.

A clinical ECG device may be used for short-term monitoring, but it is often large and the attached electrodes reduce the flexibility/mobility of the wearer. For long-term monitoring, a portable Holter monitor device can be used to detect the heart arrhythmias. Recently, wireless ECG monitoring systems are implemented [1, 2]. Bluetooth and Zigbee are the main wireless transmission protocols used in the ECG wireless monitoring systems.

The development of remote ECG Monitoring Systems (MSs) through telecommunication networks have become an important research topic. Such systems facilitate remote monitoring of people wearing portable devices equipped with Wireless Sensor Networks (WSNs) for ECG monitoring [3] as part of the Smart Home concept [4]. The ECG signals are transmitted to a local hub and then sent to an ECG server that can be used in hospitals for further analysis offering long-distance heart health monitoring. New developments in mobile devices, biosensors, and wireless equipment further the online MSs. In addition, MSs can provide automatic alerts to a physician based on the intelligent ECG processing, which in turn can assist the wearer depending on the detected anomalies.

Typically, the performance of Zigbee and Bluetooth are relatively similar, however, the use of Zigbee is more common environmental control systems and home monitoring systems. Zigbee is also currently in numerous applications including elderly people monitoring, who suffer from heart diseases and/or diabetes and other real-time 
monitoring systems. While there are many personal medical devices that use Bluetooth, this paper focuses on and emphasizes the reliability and mass-market deployment advantages of merging medical WSNs, primarily ECG monitoring, into home control networks, and thus focusses on Zigbee based home health monitoring.

Chapter II discusses home health monitoring, Chapter III presents the topic of wireless ECG monitoring and Chapter IV presents the challenges and future directions for home healthcare monitoring. The paper concludes in Chapter V.

\section{Home HeALth Monitoring Systems USING ZigBEE}

Wireless communication enabled healthcare systems offer light weight intelligent sensing at low cost, ideal for consumer mass market penetration. Recently, advancements in medical sensors, networking, and physiological data processing have enabled new devices and services. Ubiquitous health monitoring is emerging to support personal healthcare, physicians' diagnosis and to provide a first aid response in cardiac arrest cases.

\section{A. Monitoring System Architecture}

For monitoring a cardiac arrest, a portable device can be attached to the person that consists of a wireless enabled processor to transmit the sensed ECG signal from the body. Typically, the ECG signal, heartbeat, and pulse rate of the wearer are all sensed. Thus, for any detected abnormalities, action can be taken by issuing and alert message to the physician or the nearby hospital. To enable communication out of the house, to the hospital, cellular communication or an Internet connection can be used to assist the wearer in the cardiac arrest case [5].

\section{B. Mathematical Model of ECG Signals and Zigbee-based ECG Monitoring}

Typically, the sensors' characteristics can be modeled using [6]:

$$
G\left(x_{i}, \eta_{i}\right)=s\left(x_{i}, \eta_{i}\right)
$$

where $G$ is the sensor's output, $x_{i}$ is the sensing position, and $\eta_{i}$ represents the characteristic parameter. In addition, one of the applied methods is to model the physiological signals, including the ECG signal, using the Fourier transform, which can be used represented as:

$$
E C G(t, f)=\frac{1}{2 \pi}\left|\int e^{-j 2 \pi f z} b(\tau) f(\tau-t) d \tau\right|^{2}
$$

where $E C G(t, f)$ is the output sensed signal, and $f(\tau-t)$ is the sliding window function along $b(\tau)$. Afterwards, the Personal Radiation Detector (PRD) can be used to detect the abnormality difference of the person compared to 'normal' conditions or can be learnt over time. The difference between the current signal and reference signal is found, where a large PRD value potentially indicates a serious condition that the wearer may be suffering from. The processed ECG output signal is then feed to the house hub via Zigbee for further processing. Typically, the foremost objective of the Zigbee network is then to be able the house hub to have high coverage throughout the house, directly or via mesh networking. Thus, the Zigbee operation and battery characteristics models become an active research area. 


\section{Zigbee Design}

Zigbee is a typical open technology that can handle low-power/low-cost demands of the wireless networks through short-range radio. It is used mainly in Radio Frequency (RF) applications that entail long battery life, secure networking, and only requires a relatively low data rate. Zigbee mesh networking topology may provide more extensive range as well as high reliability, through the use of multiple home hubs, when available.

The Zigbee Alliance developed the Zigbee specifications for several applications in sensor networks and control for low cost connectivity and low power for devices that operate on batteries. The main Zigbee characteristics are based on the IEEE 802.15.4 standards [7]. Similar to any equipped device using batteries, Zigbee requires the sleep and shut down ability. In Zigbee nodes, the battery usage has several advantages, including increases the Zigbee device flexibility, low-cost, and batteries make Zigbee appropriate for scalable network with low duty cycle. The Zigbee devices' battery power consumption occurs through the reception/transmission time. Meanwhile, the IEEE 802.15.4 standard uses an enormously low duty cycle. Thus, in Zigbee networks, the wireless device is in sleep mode most of the time offering a long battery life, typically for many years, dependent on the device operation [8].

Generally, the power consumption is a function of the battery, duty cycle, processing, reception windows, electronic components and systems of the network, battery energy of the Zigbee network. For one node in the Zigbee network, the main energy consumption components are included in the battery total power consumption [9] as:

$$
P_{C}=P_{t}+\mathrm{P}_{r}+P_{s}+P_{i d}
$$

where, $P_{C}$ is the total power consumption, power consumption owing to the transmitted signal and the received signal are $P_{t}$ and $\mathrm{P}_{r}$ respectively. During the sleeping mode, the power consumption is represented by $P_{s}$ while $P_{i d}$ represents the power consumption due to the idle/sleep state at which zero packets are received/transmitted. The battery lifetime can be calculated in hours based on the following expression:

$$
B T=L_{c} / L^{n},
$$

where, $B T$ represents the battery life time in hours, the battery capacity is $L_{c}$ in mAH, and the load current is $L$ in $\mathrm{mA}$, where, $n$ is the Peukert's exponent ranging from 1 to 1.3. These mathematical models have been used to calculate the battery life time of the Zigbee device [10].

\section{Evaluation of Zigbee Medical Sensor Networks}

In clinical diagnosis, measuring and monitoring several physiological parameters is an essential process. Such parameters are measured using attached biomedical sensors to the person. The significant parameters to the physician include the pulse rate, blood pressure, ECG and temperature. However, these measurements are performed using analogue equipment resulting in a low degree of flexibility as the wearer is tied to the monitoring device. In addition, information sharing and backup become impossible due to the analogue equipment, where the sensors are connected to monitor devices through wires.

A potential problem arises due to wires tending to restrict mobility. Thus, for the sensor data, a wireless network system leads to greater elasticity for the user. Using computers remotely allows for personal healthcare monitoring since the wearer is coupled to a biomedical sensor network. In addition, smart signal processing techniques are used to process the sensed signal locally before communication to the hub, rather than transmitting large amounts of raw data to a hub. Interesting engineering tradeoffs exist between the required power for local processing of sensed data, vs. the wireless power required to transmit larger amounts data for remote processing. 
A typical sensor network consists of one/more Body Area Networks (BANs) and one/more interconnected hubs [11]. The received information at a hub may then be passed on via a home backbone network. Finally, the information is displayed at the connected terminals to the network [12]. This monitoring system has the prospective of instant diagnosis, ECG monitoring, and in-home monitoring. The wireless sensor nodes all run on batteries which forms the need to require power efficient wireless network systems.

The IEEE 802.15.4/Zigbee standard is considered a promising wireless standard that provides low power consumption and great flexibility for wireless networks. It offers low cost, small hardware, and can have many devices in one network.

\section{E. Operation of Sensor Nodes through Zigbee}

Unicast, broadcast, anycast, and multicast are the four data transmission methods to categorize the communication nodes. Broadcast and multi-cast are one-to-many transmission as they can convey information to numerous receivers. Nevertheless, unicast transports packets only to a single hub, thus, have less traffic overhead. On the other hand, anycast is an innovative network routing methodology wherein the information from a sender is directed to the nearest topology hub.

Sensor node operation is as follows [13]. Once a sensor unit obtains the vital signs, such as the ECG signal and heart rate, it notifies the Zigbee unit to examine the existence of a route to a hub. In the case of available route, the Zigbee unit will transmit the information packets to the hub. Else, the Zigbee unit encapsulates a message into a frame which is broadcasted to the adjacent router nodes. Regarding the data receiver, once the Zigbee unit collects the information packet, it records route in the updated routing table. In the case of collecting more than one information packet by the Zigbee unit, the extra data received may be deleted, or collected for diversity reception.

Periodically, the sensor unit can sample the vital signs/ECG signals and buffer the data. Once the buffer becomes full, the Zigbee unit creates a DATA message from the condensed data for transmission. Afterwards, periodically the Zigbee unit checks the Acknowledgement (ACK) message derived from successful reception. Once the Zigbee unit obtains an ACK message, it may delete the acknowledged data, while if within a timeout, the ACK message is not received, the Zigbee unit checks its DataReceiver list.

\section{WIRELESS ECG MONITORING SYSTEM}

Vital signal monitoring is considered the most effective procedure for remote/continuous personal health tracking. Trends in proactive healthcare are leading to the implementation of wearable sensor devices in modern tele-medicine, which in turn is enabled due to the vast progress in mobile computing, low-power microprocessors embedded with wireless technology and sensor technologies that offer accurate ubiquitous health monitoring [14]. ECG monitors are employed in several hospitals for diagnosing and monitoring the status of person's health by measuring cardiac activity. The wireless ECG MSs acquire, amplify, processes and transmit the wearer's ECG signal over wireless connection. Sensor-based incorporated systems are able to sense, process, and communicate the medical information to the involved parties, including the physicians and the emergency services' agents $[5,15]$. 


\section{A. EEG Sensors}

Recently, wearable health monitoring devices play a vital role in tele-healthcare. Such devices ranging from pulse monitors, portable ECG monitors, fall detection, activity monitors [16], through to implantable sensors. Typically, the ECG is equipped with electrodes (ECG sensors) attached to lower/upper chest to quantify the cardiac activity based on short samples of the electrical activity of the heart between the different electrode pairs. Several biomonitor systems are used to provide continuous monitoring of several physiological parameters. These systems are engaged to detect, to process and to record the signals.

An ECG signal is acquired by gauging five electrical potential between several points of the human body through an amplifier connected to the body through attached leads to electrodes [17]. Electrodes detect the electrical currents that extend through the body due cardiac cells repolarisation and depolarisation. The typical cardiac monitoring system involves 3-leads that are connected to 3-electrodes to the wearer for forming an Einthoven's triangle. Electrodes are located on different sides of the heart to measure the activity of the heart muscles as well as the voltage between the electrode pairs, which is often presented in graph form.

\section{B. ECG Communication Procedure}

Advanced monitoring systems primarily use wireless technology to connect to the sensor devices. This may form several nodes into a Wireless BAN (WBAN). Zigbee, Bluetooth, and WiFi are typically used wireless communication standards. Typically, the WLAN is used for communication in the home to the primary hub, typically Internet enabled.

Zigbee, is different than Bluetooth as it can easily be used in implementing complex home networks up to 65,536 devices. It is used in several biomedical devices implementation $[18,19]$.

\section{Personal Healthcare Monitoring Systems}

Personal healthcare monitoring systems typically involve several devices for monitoring the person's status, including ECG, vital signs and blood pressure [20]. These systems may use wireless monitoring technologies to support the healthcare. Several studies have been conducted to implement wireless monitoring systems for improving the healthcare of people [21-23]. Typically, Bluetooth, WiFi, RFID (Radio Frequency Identification) and Zigbee have been used for wireless communication. TABLE I includes a comparison between these various technologies in terms of several metrics.

TABLE I

COMPARISON BETWEEN WIRELESS TECHNOLOGY STANDARDS CHARACTERISTICS

\begin{tabular}{lll}
\hline \hline \multicolumn{1}{c}{ Symbol } & Bluetooth & Zigbee \\
\hline Battery life & Days-years & Days-years \\
Number of connected & Not defined & Up to 66,029 \\
devices & Typically $<14$ & \\
Coverage (range) & Up to 100m & Up to $75 \mathrm{~m}$ \\
Data rate & 1Mb/s & $249 \mathrm{~kb} / \mathrm{s}$ \\
Topology & (2Mb/s for BLE5) & \\
\hline \hline
\end{tabular}


In terms of data reliability including power consumption, scalability, and interference; Bluetooth has superior performance against interference compared to Zigbee and Wi-Fi. However, Bluetooth is inefficient in public congested places, including hospitals, clinics, train stations, schools, which affect the service quality of the BAN. Moreover, Zigbee has superior scalability compared to the Bluetooth as it can connect up to 65,536 sensors at a time in the BAN.

As follows, different studies that have been conducted for ECG monitoring systems based on these wireless technologies. Bluetooth-based monitoring technology has been used in several applications. Ekström [24] designed a wireless sensor structure for patient monitoring with high flexibility, free mobility, and the ECG sensor's consistency. This system includes a Personal Digital Assistant (PDA) to transmit ECG sensor's signal. Besar et al. [25] developed an ECG based MS. This monitoring system used Data Acquisition unit (DAQ), processing unit, ECG sensor, and a transceiver. Through Bluetooth, the ECG signal was transferred to the server for supplementary analysis.

Moreover, the WiFi-based monitoring technology has been employed is several applications. A wireless monitoring system has been developed by $\mathrm{Yu}$ and Cheng [26] based on Bluetooth and WiFi. It was designated to obtain the wearer's physiological signals for further transmitting them through Bluetooth to a server. A control center, mobile devices, monitor unit, and a web page communicated through WiFi. In order to increase mobility, Bluetooth may be combined with WiFi networks. Noimanee et al. [27] designed a monitoring system that included a video camera, an ECG transceiver to send the biosignals through Zigbee/IEEE RF unit, and a WiMAX transceiver to send the ECG signals. In the case of detecting an ECG abnormality, the ECG sensor will direct the signals to the video camera that is near to the patient to be monitored by physicians. Of course, physicians may not be available at the time.

RFID-based monitoring technology also has a significant role in home healthcare. Ishihata et al. [28] proposed an implantable RFID chip to store the person's information and to track the location. Trappey et al. [29] proposed a RFID-based mobile intelligent medical system that consisted of location broadcasting RFID application and an identification system to reduce the data entry redundancy/error, and to provide intelligent decision making. Teaw et al. [30] developed a wireless sensor structure for monitoring of vital signs, such as heart rate, respiration rate, blood pressure, and body temperature. In addition, the system tracked the patient's location through RFID tags to alert their relatives when required. A bar code printed on the wrist bands can be used to monitor the patients in hospitals. In addition, Want [31] proposed to replace the bar codes by RFID tags to give the ability to store more information. For real-time monitoring, Ogawa et al. [32] designed a body matched tags. Such tags have been used also for patients' localization. Cho et al. [33] extended the network area coverage for active RFID devices though a mesh type multihop network, while using Zigbee between the hubs and the RFID readers.

Frehill et al. [34] proposed a new architecture to measure the patient's signs through a friendly user Interface. Wireless ECG and Pulse-Oximeter sensors were included to share the information. The results established the efficiency of Zigbee to meet achieve the required mobility, power, and data throughput. Park et al. [35] implemented a Zigbee Network-based Multi-channel Heart Rate MS for exercise rehabilitation patients. This monitoring system involves a central MS and a wearable Patient's Side Device (PSD). The central MS can simultaneously monitor several patients. A warning signal is generated if necessary and linked with the MS through the Zigbee network. Becher et al. [36] designed a Zigbee based system along with Bluetooth to incorporate the patient's ECG, body weight, and pulse. Three cardiovascular wireless sensors are involved to monitor the ECG signal, body weight and pulse. The patient's information is gathered from these sensors through a microcontroller for further data processing which is forwarded to a Zigbee gateway. The Zigbee gateway was used to forward the packets through a Bluetooth transceiver for data analysis.

While many people suffering from medical conditions are housebound, there are medical benefits from being able to go outside. Wang et al [37] presented a Zigbee based home healthcare monitoring wearable system using ECG, fall detection ad GPS to monitor people outside. 
Home healthcare systems can infer the activity of the person through their location in the home. Yan et al. [38] developed an e-health monitoring system based on multisensory data fusion to predict activities and further the decision making process concerning the person's health.

\section{HOME CONTROL AND SMART-HOME SERVICES}

Zigbee has been used as the wireless communication protocol for home automation systems, including smart energy control [39-41], home control [42] and environmental monitoring [43]. More recently, the smart home concept [4] has furthered the services to consumers offering accessible displays [44], remote device control [45, 46], and device collaboration [47, 48], through always-on Internet home gateways [49]. Interestingly, the smart-home does enable the control of humanoid-type robots for home automation and perhaps the care of the elderly using robots [50].

\section{Challenges AND Future Directions}

Many personal healthcare devices use different wireless technologies, do not have compatible interfaces and often require cable connection. Consequently, it is essential to remove wired connections and develop short-range wireless connections for feasible connectivity between devices. Zigbee over IEEE 802.15.4 and Bluetooth over IEEE 802.15.1 are the two main low power consumption wireless communications that have short range communication protocols to replace cables. Bluetooth is an inexpensive device for short range communication that based on a wireless radio system. While mesh networking is possible, it has primarily been used for point to point communication and to produce small ad-hoc networks. Bluetooth is robust, requires low complexity, low power and low cost [51, 52].

A Zigbee device can offer consistent mesh networking, multi-hop, and self-organization which complements the application software layers quantified by the IEEE 802.15 and Zigbee alliance. Zigbee can provide low power connectivity and low cost for equipment that requires long battery life. However, it does not necessitate high data transfer rates as those empowered by Bluetooth. Zigbee amenable wireless devices can be used to transmit 10-75 meters based on the applications power output consumption requirements and the RF environment [53].

However, up till now, the Zigbee current standards have not considered the reliability of transmitted messages in a multi-hop topology. Thus, Zigbee could be unsuitable for vital signs transmission, particularly, for emergency information messages, including the ECG signal. Especially, these messages are precarious for detecting the patients' disease and for offering significant clues to the insistence level. Consequently, this can be considered as an active research area.

In addition, the energy requests are founded on the application activities that can be proposed as a model to extend battery life time [54]. Additionally, other research studies are directed to improve and optimize the energy of different Zigbee routing procedures.

Developing new protocol for fast rerouting is a challenging research area. An incomplete path could be improved in a short latency to ensure the transmitted vital signs reliability. Consequently, a Zigbee-based unit can be implemented for ECG signal monitoring system using the new routing protocol.

Applying Zigbee devices in healthcare monitoring systems attracts much research. The readings and the ECG signal obtained using the Zigbee wireless network should be compared in contradiction to collected data gathered from static devices, including a thermostat, or a stethoscope for measuring the heartbeat. Nevertheless, the main limitation was the Zigbee transmission/ reception range.

Employing soft computing techniques to develop an integrated Zigbee healthcare platform in ECG monitoring can be considered as a novel active domain. This monitoring system permits patients to move while the monitoring process 
and vital signs readings are also recorded. This platform can be supplemented by using a fuzzy logic based diagnosis system. Further research can be directed to ubiquities vital signs processing and monitoring toward portable and intelligent vital signs processing [55].

Research and development has created intelligent smart homes offering a reliable connection to the Internet and cloud services, which also creating home based health monitoring systems, but this paper has presented that the two systems are sadly invariably developed separately. For health monitoring and intervention to be truly ubiquitous and cost-effective, this paper calls for health monitoring to be considered as an integral part of the overall smart home architecture, rather than a separate or as added feature. Such integration will maximize the performance, the reliability and security of the home healthcare monitoring system through the existing home hubs.

\section{CONCLUSION}

ECG is useful method for monitoring the heart electrical activity using an electrode array placed at specific positions on the body. ECG monitoring systems support a physician for understanding the patient's condition. Recently, low power, low cost, and flexible Zigbee wireless units have been deployed to sense and to transmit the remote patient ECG signal and vital signs.

This paper has considered the current state of the art in home ECG monitoring, and has noticed that such systems tended to be designed in isolation, as part of their own network, rather than part of more reliable home control systems. This paper proposes that for true mass-market consumer penetration, such home healthcare monitoring systems need to be part of a wider home control system strategy. Such a deployment strategy offers significant benefits in cost reduction through redundancy, improved device integration, reliability and latency of passing important messages.

\section{REFERENCES}

[1] M. Hong, Z. Yajun and H. Xiaoping, "Portable ECG measurement device based on MSP430 MCU," in Proc. $B M E I$, 2008 , pp. 667-671.

[2] N. Ebrahim, M. J. Deen and T. Mondal, "A wireless wearable ECG sensor for long-term applications," IEEE Commun. Mag., vol. 50, no. 1, pp. 36-43, Jan. 2012.

[3] J. M. Cano-Garcia, E. Gonzalez-Parada, V. Alarcon-Collantes and E. Casilari-Perez, "A PDA-based portable wireless ECG monitor for medical personal area networks," in Proc. MELECON, 2006, pp. 713-716.

[4] T. K. L. Hui, R. S. Sherratt and D. Diaz Sanchez, "Major requirements for building Smart Homes in Smart Cities based on Internet of Things technologies," Future Generation Computer Systems, vol. 76, pp. 358-369, Nov. 2017.

[5] A. Pantelopoulos and N. G. Bourbakis, "A survey on wearable sensor-based systems for health monitoring and prognosis,” IEEE Trans. Syst. Man, Cybern. B., vol. 40, no. 1, pp. 1-12, Jan. 2010.

[6] V. R. Sarma Dhulipala, and G. R. Kanagachidambaresan, "Cardiac care assistance using self configured sensor network - a remote patient monitoring system," J. Institution of Engineers India Series B, vol. 95, no. 2, pp. 101-106, April. 2014.

[7] C.E. Sinem. (2004, Sept.). ZigBee/IEEE 802.15. 4 Summary. [on-line]. Available: https://www.researchgate.net/publication/242712090_ZigBeeIEEE_802154_Summary

[8] S. F. Qadri, S. A. Awan, M. Amjad, M. Anwar and S. Shehzad, "Applications, challenges, security of wireless body area networks (WBANs) and functionality of IEEE 802.15.4/ZIGBEE,” Sci. Int.(Lahore), vol. 25, no. 4, pp. 697-702, 2013.

[9] A. Hussein and G. Samara, "Mathematical modeling and analysis of zigbee node battery characteristics and operation," MAGNT Research Report, vol. 3, no. 6, pp. 99-106, 2015. 
[10] R. Srinivasan, D. Z. Turker, S. W. Park and E. Sánchez-Sinencio, "A low-power frequency synthesizer with quadrature signal generation for $2.4 \mathrm{GHz}$ zigbee transceiver applications," in Proc. ISCAS, 2007, pp. 429-432.

[11] A. Ghamari, B. Janko, S. Sherratt, W. Harwin, R. Piechockic and C. Soltanpur, "A survey on wireless body area networks for eHealthcare systems in residential environments," Sensors, vol. 16, no. 16, 831, Jun. 2016.

[12] K. Øyri, I. Balasingham, E. Samset, J. O. Høgetveit and E. Fosse, "Wireless continuous arterial blood pressure monitoring during surgery: a pilot study," Anesthesia \& Analgesia, vol. 102, no. 2, pp. 478-483, Feb. 2006.

[13] V. M. Bondre, "Review of Health Monitoring System Using Zigbee Based Variable Devices," Int. J. Scientific Engineering and Applied Science, vol. 1, no. 7, pp. 174-177, Oct. 2015.

[14] R. C. King, E. Villeneuve, R. J. White, R. S. Sherratt, W. Holderbaum and W. S. Harwin, "Application of data fusion techniques and technologies for wearable health monitoring," Medical Engineering \& Physics, vol. 42, pp. 1-12, Apr. 2017.

[15] J. Wang, Z. Zhang, B. Li, S. Lee and R. Sherratt, "An Enhanced Fall Detection System for Elderly Person Monitoring using Consumer Home Networks," IEEE Trans. Consum. Electron., vol. 60, no. 1, pp. 23-29, Feb. 2014.

[16] Z. A. Khan and W. Sohn, "Abnormal Human Activity Recognition System Based on R-Transform and Kernel Discriminant Technique for Elderly Home Care," IEEE Trans. Consum. Electron., vol. 57, no. 4, pp. 18431850, Feb. 2011.

[17] P. J. Xu, H. Zhang and X. M. Tao, "Textile-structured electrodes for electrocardiogram," Textile Progress, vol. 40, no. 4, pp. 183-213, Dec. 2008.

[18] H. J. Lee, S. H. Lee, K. Ha, H. C. Jang, W. Chung, J. Y. Kim, Y. Chang and D. Hyun Yoo, "Ubiquitous healthcare service using Zigbee and mobile phone for elderly patients," Int. J. Medical Informatics, vol. 78, no. 3, pp. 193-198, Mar. 2009.

[19] T. Gao, C. Pesto, L. Selavo, Y. Chen, J. Ko, J. Lim, A. Terzis, A. Watt, J. Jeng, B. Chen, K. Lorincz and M. Welsh, "Wireless medical sensor networks in emergency response: Implementation and pilot results," in Proc. THS, 2008, pp. 187-192.

[20] C.-H. Hung, Y.-W. Bai and R.-Y. Tsai, "Design of Blood Pressure Measurement with a Health Management System for the Aged," IEEE Trans. Consum. Electron., vol. 58, no. 2, pp. 619-625, May 2012.

[21] K.-A. Kim, S.-Y. Shin, J.-W. Suh, C. Park, E.-J. Cha and H.-D. Bae, "Home healthcare self-monitoring system for chronic diseases," in Proc. ICCE, 2012, pp. 486-487.

[22] W.-J. Li, Y.-L. Luo, Y.-S. Chang and Y.-H. Lin, "A wireless blood pressure monitoring system for personal health management," in Proc. EMBC, 2010, pp. 2196-2199.

[23] H. Y. Tung, K. F. Tsang, H. C. Tung, K. T. Chui and H. R. Chi, "The Design of Dual Radio ZigBee Homecare Gateway for Remote Patient Monitoring," IEEE Trans. Consum. Electron., vol. 59, no. 4, pp. 756-764, Nov. 2013.

[24] M. Ekström, "Small wireless ECG with Bluetooth ${ }^{\mathrm{TM}}$ communication to a PDA," MS. dissertation, Mälardalen University, Sweden, 2006.

[25] R. Besar, Y. S. Tan, K. H. Tee and K. C. Ong, "Bluetooth-enabled ECG monitoring system," in Proc. TENCON, 2005, pp. 1-5.

[26] S.-N. Yu and J.-C. Cheng, "A wireless physiological signal monitoring system with integrated bluetooth and WiFi technologies," in Proc. EMBC, 2005, pp. 2203-2206.

[27] K. Noimanee, S. Noimanee, P. Khunja and P. Keawfoonrungsie, "Medical consult-based system for diagnosis on WiMAX technology," I. J. Applied Biomedical Engineering, vol. 3, no. 1, pp. 51-55, 2010.

[28] H. Ishihata, T. Tomoe, K. Takei, T. Hirano, K.Yoshida, S. Shoji, H. Shimauchi and H. Horiuchi, "A radio frequency identification implanted in a tooth can communicate with the outside world," IEEE Trans. Inf. Technol. Biomed., vol. 11, no. 6, pp. 683-685, Nov. 2007.

[29] C. V. Trappey, C. S. Liu and A. J. C. Trappey, "Develop patient monitoring and support system using mobile communication and intelligent reasoning," in Proc. SMC, 2009, pp. 1195-1200.

[30] E. Teaw, G. Hou, M. Gouzman, K. W. Tang, A. Kesluk, M. Kane and J. Farrell, "A wireless health monitoring system," in Proc. IA, 2005, pp. 247-252.

[31] R. Want, "An introduction to RFID technology," IEEE Pervasive Comp., vol. 5, no. 1, pp. 25-33, Feb. 2006.

[32] H. Ogawa, Y. Yonezawa, H. Maki, I. Ninomiya, K. Sata, S.Hamada and W. M. Caldwell, "A new infusion pathway intactness monitoring system," in Proc. EMBC, 2006, pp. 4999-5000. 
[33] H. Cho, J. Kim and Y. Baek, "Large-Scale Active RFID System Utilizing ZigBee Networks," IEEE Trans. Consum. Electron., vol. 57, no. 2, pp. 379-385, May. 2011.

[34] P. Frehill, D. Chambers and C. Rotariu, "Using zigbee to integrate medical devices," in Proc. EMBC, 2007, pp. 6171-6720.

[35] J. Park, J. Cho, J. Choi and T. Nam, "A zigbee network-based multi-channel heart rate monitoring system for exercising rehabilitation patients," in Proc. TENCON, 2007, pp. 1-4.

[36] K. Becher, C. P. Figueiredo, C. Mühle, R. Ruff, P. M. Mendes and K.-P. Hoffmann, "Design and realization of a wireless sensor gateway for health monitoring," in Proc. EMBC, 2010, pp. 374-377.

[37] L.-H. Wang, Y.-M. Hsiao, X.-Q. Xie and S.-Y. Lee, "An Outdoor Intelligent Healthcare Monitoring Device for the Elderly," IEEE Trans. Consum. Electron., vol. 62, no. 2, pp. 128-135, May 2016.

[38] H. Yan, H. Huo, Y. Xu and Mikael Gidlund, "Wireless Sensor Network Based E-Health System Implementation and Experimental Results," IEEE Trans. Consum. Electron., vol. 56, no. 4, pp. 2288-2295, Nov. 2010.

[39] J. Han, C.-S. Choi, W.-Ki Park, I. Lee and S.-Ha Kim, "Smart Home Energy Management System Including Renewable Energy Based on ZigBee and PLC," IEEE Trans. Consum. Electron., vol. 60, no. 2, pp. 198-202, May 2014.

[40] D.-M Han and J.-H. Lim, "Design and Implementation of Smart Home Energy Management Systems based on ZigBee," IEEE Trans. Consum. Electron., vol. 56, no. 3, pp. 1417-1425, Aug. 2010.

[41] D.-M Han and J.-H. Lim, "Smart Home Energy Management System using IEEE 802.15.4 and ZigBee," IEEE Trans. Consum. Electron., vol. 56, no. 3, pp. 1403-1410, Aug. 2010.

[42] N. Kushiro, T. Higuma, M. Nakata, H. Kubota and K. Sato, "Practical Solution for Constructing Ubiquitous Network in Building and Home Control System," IEEE Trans. Consum. Electron., vol. 53, no. 4, pp. 13971392, Nov. 2007.

[43] J. Byun, B. Jeon, J. Noh, Y. Kim and S. Park, "An Intelligent Self-Adjusting Sensor for Smart Home Services based on ZigBee communications,” IEEE Trans. Consum. Electron., vol. 58, no. 3, pp. 794-802, Aug. 2012.

[44] L. C. P. Costa, N. S. Almeida, A. G. D. Correa, R. D. Lopes and M. K. Zuffo, "Accessible Display Design to Control Home Area Networks," IEEE Trans. Consum. Electron., vol. 59, no. 2, pp. 422-427, May 2013.

[45] I. A. Zualkernan, A. R. Al-ali, M. A. Jabbar, I. Zabalawi and Ahmed Wasfy, "InfoPods: Zigbee-Based Remote Information Monitoring Devices for Smart-Homes," IEEE Trans. Consum. Electron., vol. 55, no. 3, pp. 12211226, Aug. 2009.

[46] A. Sleman and R. Moeller, "SOA Distributed Operating System for Managing Embedded Devices in Home and Building Automation," IEEE Trans. Consum. Electron., vol. 57, no. 2, pp. 945-952 May 2011.

[47] H. Park, I. Lee, T. Hwang and N. Kim, "Architecture of home gateway for device collaboration in extended home space," IEEE Trans. Consum. Electron., vol. 54, no. 4, pp. 1692-1697, Nov. 2008.

[48] T. Perumal, A. R. Ramli and C. Y. Leong, "Interoperability Framework for Smart Home Systems," IEEE Trans. Consum. Electron., vol. 57, no. 4, pp. 1607-1611, Nov. 2011.

[49] W.-K. Park, C.-Sic Choi, I.-W. Lee and J. Jang, "Energy Efficient Multi-Function Home Gateway in AlwaysOn Home Environment," IEEE Trans. Consum. Electron., vol. 56, no. 1, pp. 106-111, Feb. 2012.

[50] K. Kim, Y.-S. Cha, J.-M. Park, J.-Y. Lee and B.-J. You, "Providing Services Using Network-Based Humanoids in a Home Environment," IEEE Trans. Consum. Electron., vol. 57, no. 4, pp. 1628-1636, Nov. 2011.

[51] M. Petrova, J. Riihijarvi, P. Mahonen, and S. Labella, "Performance study of IEEE 802.15.4 using measurements and simulations," in Proc. WCNC, 2006, pp. 487-492.

[52] X. Fafoutis, B. Janko, F. Mellios, G. Hilton, S. Sherratt, R. Piechocki and I. Craddock, "SPW-1: A LowMaintenance Wearable Activity Tracker for Residential Monitoring and Healthcare Applications," eHealth $360^{\circ}$. Lecture Notes of the Institute for Computer Sciences, Social Informatics and Telecommunications Engineering, vol. 181, pp. 294-305, 2017

[53] M. M. Kochhal, "Unified role assignment framework for wireless sensor networks," Ph.D. dissertation, Wayne State University, MI, 2010.

[54] H. Arora, R. S. Sherratt, B. Janko and W. S. Harwin, "Experimental Validation of the Recovery Effect in Batteries for Wearable Sensors and Healthcare Devices," IET J. Engineering,

[55] D. G. Guo, F. E. H. Tay, L. Xu, L. M. Yu, M. N. Nyan, F. W. Chong, K. L. Yap and B. Xu, "A long-term wearable vital signs monitoring system using BSN," in Proc. EUROMICRO, 2008, pp. 825-830. 


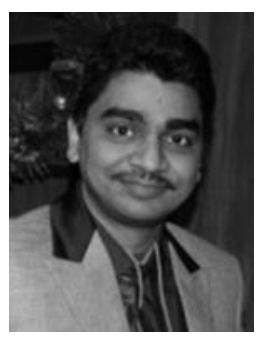

Nilanjan Day was born in Kolkata, India, in 1984. He received his B.Tech. degree in Information Technology from West Bengal University of Technology in 2005, M.Tech. in Information Technology in 2011 from the same University and Ph.D. in digital image processing in 2015 from Jadavpur University, India.

In 2011, he was appointed as an Asst. Professor in the Department of Information Technology at JIS College of Engineering, Kalyani, India followed by Bengal College of Engineering College, Durgapur, India in 2014. He is now employed as an Asst. Professor in Department of Information Technology, Techno India College of Technology, India. His research topic is signal processing, machine learning and information security.

Dr. Dey is an Associate Editor of IEEE ACCESS and is currently the Editor in-Chief of the International Journal of Ambient Computing and Intelligence, International Journal of Rough Sets and Data Analysis, International Journal of Natural Computing Research, Co-Editor-in-Chief of International Journal of Synthetic Emotion and Series Editor of Advances in Geospatial Technologies Book Series.

Amira S. Ashour was born in Tanta, Egypt, in 1975. She is graduated from Faculty of Engineering, Tanta University, Egypt in 1997. She received her Master degree in Electrical Engineering in 2001from the same university and $\mathrm{Ph} . \mathrm{D}$. in smart antenna in 2005 from the Department of Electronics and Electrical Communications Engineering, Faculty of Engineering, Tanta University, Egypt.

In 2005, she was appointed as a lecturer in the Department of Electronics and Electrical Communications Engineering, Faculty of Engineering, Tanta University, Egypt. She was the Vice Chair of CS department, CIT College, Taif University, KSA from 2009 till 2015. She was the Vice Chair of Computer Engineering Department, Computers and Information Technology College, Taif University, KSA for one year in 2015. She is now employed as an Assistant Professor and Head of Department in Department of Electronics and Electrical Communications Engineering, Faculty of Engineering, Tanta University, Egypt. Her research topics are smart antenna, direction of arrival estimation, targets tracking, image processing, medical imaging, machine learning, and image analysis.

Dr. Ashour is an Editor-in-Chief for the International Journal of Synthetic Emotions. She is an Associate Editor for the International Journal of Rough Sets and Data Analysis, as well as the International Journal of Ambient Computing and Intelligence. She is an Editorial Board Member of the International Journal of Image Mining. She published over 80 journal papers and conference proceedings.

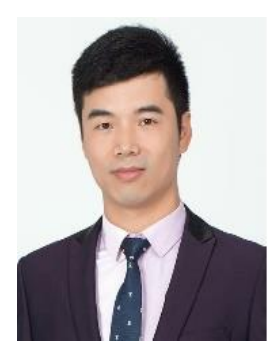

Fuqian Shi (M'08-SM'10) was born in Wenzhou, China in 1975. He received the Bachelor degree in Wenzhou University, China in 1997, M.Sc. in Zhejiang University of Technology in 2006 and Ph.D. in 2011 from Zhejiang University, China.

$\mathrm{He}$ is currently an Associate Professor at College of Information and Engineering, Wenzhou Medical University, China. He was invited as Visiting Associate Professor in the Department of Industrial Engineering and Management Systems, University of Central Florida, USA from 2012 to 2014. His research interests include biomedical engineering, fuzzy inference system, and artificial neuro networks.

Dr. Shi has served on over 20 international committee conference board memberships, and serves as an Associate Editor of the International Journal of Ambient Computing and Intelligence and International Journal of Rough Sets and Data Analysis. Dr. Shi is a reviewer of IEEE TRANSACTIONS ON FUZZY SYSTEMS, IEEE TRANSACTIONS ON SYSTEMS, MAN AND CYBERNETICS, Information Sciences, etc. He has published over 50 journal papers and conference proceedings.

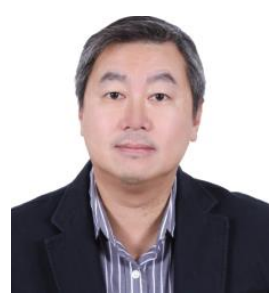

Simon Fong was graduated from La Trobe University, Australia, with a 1st Class Honours BEng. He received the Master degree in Computer Systems degree in 1993, and his PhD. in 1998.

Simon is currently an Associate Professor at the Computer and Information Science Department of the University of Macau. He is a co-founder of the Data Analytics and Collaborative Computing Research Group in the Faculty of Science and Technology. Prior to his academic career, Simon took up various managerial and technical posts, such as systems engineer, IT consultant and ecommerce director in Australia and Asia. 
Dr. Fong serves on the editorial boards of the Journal of Network and Computer Applications by Elsevier, IEEE IT PROFESSIONAL MAGAZINE, and various special issues of SCIE-indexed journals. He has published over 380 international conference and peer-reviewed journal papers, mostly in the areas of data mining, data stream mining, big data analytics, meta-heuristics optimization algorithms, and their applications.

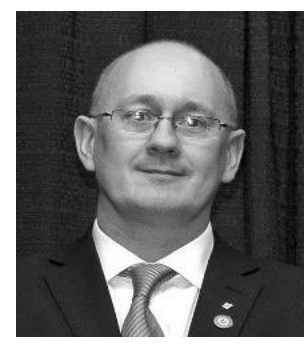

R. Simon Sherratt (M'97-SM'02-F'12) was born near Liverpool, England, in 1969. He received the B.Eng. degree in Electronic Systems and Control Engineering from Sheffield City Polytechnic, UK in 1992, M.Sc. in Data Telecommunications in 1994 and Ph.D. in video signal processing in 1996 from the University of Salford, UK.

In 1996, he has appointed as a Lecturer in Electronic Engineering at the University of Reading where he is now Professor of Biosensors in the Department of Biomedical Engineering. His research topic is signal processing and personal communications in consumer devices focusing on wearable devices and healthcare.

Professor Sherratt received the $1^{\text {st }}$ place IEEE Chester Sall Memorial Award in 2006, the $2^{\text {nd }}$ place in 2016 and the $3^{\text {rd }}$ place in 2017. He is a reviewer for the IEEE SENSORS JOURNAL on wearable devices and is currently Emeritus Editor in-Chief of the IEEE TRANSACTIONS ON CONSUMER ELECTRONICS. 